Monthly SSB imports increased by 369000 litres per month following the tax [95\% CI 325,000 to 413 000]. Non-SSB imports increased by 281000 litres per month [95\% CI 268,000 to 294, 000].

Conclusion The Barbados SSB tax was not associated with a reduction in SSB sales at this grocery store chain, although there is evidence of a reduction at the end of the study period. National data suggest that SSB imports (which may be cheaper) increased. The grocery store analysis also suggest that sales of imported SSBs increased, while locally manufactured SSBs decreased, suggesting that consumers may be substituting to imported beverages. A higher rate of tax may be needed to reduce consumption of SSBs.

\section{OP5 \#EXPLORING INDUSTRY INFLUENCE ACROSS ALCOHOL AND SUGAR PRICING POLICIES IN THE UK: A DISCOURSE NETWORK ANALYSIS}

S Hilton*, C Buckton, G Fergie. MRC/CSO SPHSU, University of Glasgow, Glasgow, UK

\subsection{6/jech-2018-SSMabstracts.5}

Background Increasingly unhealthy commodity industries (UCIs) strategically use the news media to influence public opinion and the political agenda in favour of advancing their preferred policy options. In politically-charged pricing policy debates, such as Minimum Unit Pricing (MUP) for alcohol and sugar-sweetened beverage (SSB) taxation industry efforts to disrupt the introduction of these pricing policies has been significant. By comparing MUP for alcohol and SSB taxation media debates using discourse network analysis (DNA) we aim to visually map the actors and their relationships highlighting similarities and differences across industry sectors.

Methods Eleven national UK newspapers, spanning political views and genre, were searched using the Nexis database between May 2011 and November 2012 to identify all published articles relating to alcohol and pricing, and between May 2015 and November 2016 to identify all published articles relating to sugar/beverage and tax/levy. Statements made by actors and organisations in the debates were inductively identified and coded using network analysis software to produce relational data to generate visualisations of discourse networks.

Results For MUP for alcohol 1435 statements made by 151 individuals from 87 organisations were coded in 351 articles. For SSB taxation 3882 statements made by 214 individuals from 177 organisations were coded in 511 articles. The construction of MUP for alcohol and SSB taxation networks provides the first visual evidence of the positioning of industry representatives across two policy debates.Both networks show tight discourse coalitions of manufactures acting in opposition to policy advocates, with the largest corporations most active, and most central in these coalitions at key points in the debate. Less active industry representatives and cross-sector corporations (such as supermarkets) are more peripheral to the network, indicating both cleavages within industries and across corporate actors.

Conclusion By comparing the discourse networks across two highly contested pricing policy debates, we have visualised the complex network of actors and relationships operating to directly influence pricing policy-making via the media. Conducting comparative discourse network analysis across policy debates shows promise for better understanding the common tactics of different UCIs to disrupt public health policies. This is important for supporting public health advocates to develop more effective media advocacy strategies for exposing and opposing UCI tactics and strategies and in identifying public health messages which might be targeted to generate public and policy support for pricing policies.

\section{Obesity}

\section{OP6 RAPID WEIGHT GAIN IN THE FIRST FEW YEARS OF LIFE AND CHILD-TO-ADOLESCENCE BMI TRAJECTORIES: THE UK MILLENNIUM COHORT STUDY}

${ }^{1} Y L_{L}{ }^{*},{ }^{2} \mathrm{~A}$ Pearce, ${ }^{1} \mathrm{~L} \mathrm{Li} .{ }^{1}$ GOS Institute of Child Health, UCL, London, UK: ${ }^{2} \mathrm{MRC} / \mathrm{CSO}$ Social and Public Health Sciences Unit, University of Glasgow, Glasgow, UK

\subsection{6/jech-2018-SSMabstracts.6}

Background High birthweight and rapid weight gain (RWG) in early life are independently and positively associated with obesity in childhood and adulthood. It is unclear whether RWG can affect BMI growth from childhood to adolescence. RWG is common among low-birth-weight infants, especially following intrauterine growth retardation. However, whether the association between RWG and BMI trajectory differs by birthweight groups is not well understood. We aimed to investigate the effect of RWG (birth-3 y) on BMI trajectory (5-14 y) and whether associations differed by birthweight groups.

Methods We used data from the Millennium Cohort Study, a representative sample of children born in the UK in 2000-2002. We included term singletons with available information on weight gain (birth-3 y) and $1+$ BMI measurement between $5 \mathrm{y}$ and $14 \mathrm{y}(\mathrm{n}=11,522)$. Weight at birth and $3 \mathrm{y}$ were converted to internal standard deviations scores (SDS). RWG was defined as change in weight SDS from birth to $3 \mathrm{y}>0.67 \mathrm{SDS}$, which is commonly used and represents the width of each percentile band on standard growth charts. Mixed effects fractional polynomial models were applied to estimate the effects of RWG on BMI trajectories, for boys and girls separately, before and after adjustment for maternal pre-pregnancy, maternal smoking during pregnancy, parity, ethnicity, breastfeeding, early introduction to solid foods, maternal education, and family income. The analysis was further stratified by low birthweight (LBW) status.

Results Boys and girls who experienced RWG were heavier than their non-RWG counterparts at $5 \mathrm{y}$ by $1.21 \mathrm{~kg} / \mathrm{m}^{2}$ [95\% CI 1.10 to 1.33 ] and $1.25 \mathrm{~kg} / \mathrm{m}^{2}$ [1.13-1.38] respectively; continued to gain weight more rapidly between $5 \mathrm{y}$ and $14 \mathrm{y}$, especially in childhood $\left(5-7\right.$ y) by $0.2-0.3 \mathrm{~kg} / \mathrm{m}^{2}$ per year. These differences persisted after adjustment for potential confounders. The effect of RWG on BMI was particularly higher among non-LWB children, whose estimated BMI trajectories exceeded the International Obesity Task Force overweight reference lines. For example, at $14 \mathrm{y}$ the estimated mean BMI was $24.41 \mathrm{~kg} / \mathrm{m}^{2}$ [23.91-24.45] for non-LBW RWG girls (vs. $22.07 \mathrm{~kg} / \mathrm{m}^{2}$ for LWB RWG girls), exceeding the gender- and age-specific cut-off point of $23.34 \mathrm{~kg} / \mathrm{m}^{2}$ for overweight. Sensitivity analysis using 3-category birthweight variable (low/normal/high) showed that the trajectories of high-birthweight children were similar to those of normalbirthweight children. 
Conclusion RWG from birth to $3 \mathrm{y}$ was positively associated with BMI in early childhood and adiposity growth throughout childhood to adolescence. This positive effect was particularly detrimental for non-LBW than LBW children, which may have important public health implications for preventing early life RWG among term appropriate- and large-for-gestational-age children.

\section{OP7 BARRIERS AND FACILITATORS TO ADOPTION, IMPLEMENTATION AND SUSTAINMENT OF OBESITY PREVENTION INTERVENTIONS IN SCHOOLCHILDREN- A DEDIPAC CASE STUDY}

${ }^{1} \mathrm{CB}$ Hayes*, ${ }^{1} \mathrm{MP}$ O'Shea, ${ }^{2,3} \mathrm{C}$ Foley-Nolan, ${ }^{4} \mathrm{M}$ McCarthy, ${ }^{3} \mathrm{~J}$ Harrington. ${ }^{1}$ Institute of Population Health, School of Medicine, Trinity College Dublin, Dublin, Ireland; ' 2 Safefood, Cork, Ireland; ${ }^{3}$ School of Public Health, University College Cork, Cork, Ireland; ${ }^{4}$ Cork University Business School, University College Cork, Cork, Ireland

\subsection{6/jech-2018-SSMabstracts.7}

Background The aim of the study was to explore the implementation of school based diet and physical activity interventions with respect to the barriers and facilitators to adoption, implementation and sustainability; supportive actions required for implementation and recommendations to overcome identified barriers. Two interventions rolled out nationally in Ireland were chosen; Food Dudes, a programme to encourage primary school children to consume more fruit and vegetables and an Active Travel to School Programme in primary and secondary schools. Trained school coordinators (teachers) cascade the programmes to other teaching staff.

Methods Multiple case study design using qualitative semistructured interviews with key stakeholders: primary and secondary school teachers, school coordinators, project coordinators/managers, funders and intermediaries. Fifteen interviews were conducted. Data were coded using a common categorization matrix. Thematic analysis was undertaken using the Adoption, Implementation and Maintenance elements of the RE-AIM implementation framework.

Results Good working relationships within and across government departments, intermediaries and schools were critical for intervention adoption, successful implementation and sustainability. Organisational and leadership ability of coordinators was essential. Provision of participation incentives acted as motivators to engage children's interest. A deep understanding of the lives of the target children was an important contextual factor. The importance of adaptation without compromising core components in enhancing intervention sustainability emerged. Successful implementation was hindered by: funding insecurity, school timetable constraints, broad rather than specific intervention core components, and lack of agreement on conduct of programme evaluation. Supportive actions for maintenance included ongoing political support, secure funding and pre-existing healthy lifestyle policies.

Conclusion Successful implementation and scale up of public health anti-obesity interventions in schools is dependent on contextual factors, engagement and leadership at multiple levels and secure funding. Recommendations to overcome barriers include: capacity to deliver within an already overcrowded curriculum and clear specification of intervention components within a conceptual framework to facilitate evaluation. Our findings are generalisable across different contexts and are highly relevant to those involved in the development or adaptation, organisation or execution of national public health interventions; policy makers, guidelines developers, and staff involved in local organisation and delivery.

\section{OP8 ARE CLINICALLY OBESE CHILDREN AT INCREASED RISK OF HOSPITAL ADMISSION? A LONGITUDINAL ANALYSIS USING LINKED ELECTRONIC HEALTH RECORDS IN WALES AND SCOTLAND}

${ }^{1} \mathrm{U}$ Griffiths ${ }^{*},{ }^{1} \mathrm{M}$ Cortina-Borja, ${ }^{2} \mathrm{~A}$ Bandyopadhyay, ${ }^{2} \mathrm{~K}$ Tingay, ${ }^{1} \mathrm{BL}$ De Stavola, ${ }^{1} \mathrm{H}$ Bedford, ${ }^{3} \mathrm{~A}$ Akbari, ${ }^{3} \mathrm{R}$ Lyons, ${ }^{4} \mathrm{C}$ Dezateux. ${ }^{1}$ Population, Policy and Practice Programme, UCL Great Ormond Street Institute of Child Health, London, UK; ${ }^{2}$ Swansea University Medical School, Swansea University, Swansea, UK; ${ }^{3}$ Farr Institute, Swansea University Medical School, Swansea University, Swansea, UK; ${ }^{4}$ Centre for Primary Care and Public Health, Barts and the London School of Medicine and Dentistry, Queen Mary University London, London, UK

\subsection{6/jech-2018-SSMabstracts.8}

Background Few studies have examined the extent and nature of health service utilisation by obese and overweight children. We estimated the rate of hospital admission from five to 13.9 years of age in children who were clinically overweight or obese by age five years compared with their healthy weight peers, using linked electronic health records from a nationally representative prospective cohort of children in Wales and Scotland.

Methods Data were available for 3306 singleton children participating in the Millennium Cohort Study (MCS), who were living in Wales $(n=1840)$ or Scotland $(n=1466)$, and whose parents consented to linkage to their health records up until their 14th birthday. Height and weight were measured at home interview at age five years and weight status was categorised according to the UK1990 clinical reference standards. Overall, 3269 (1678 boys) of these 3306 (98.9\%) children were linked to the Welsh Demographic Service $(n=1838)$ or their Scottish Community Health Index number $(n=1431)$ via probabilistic and deterministic record matching. In turn, they were matched to Welsh and Scottish hospital admission records from the Patient Episode Database for Wales, and Scottish Morbidity Records, respectively. Using information on admissions from five to 14 years, we fitted negative binomial models adjusted by potential confounders in order to compare rates of inpatient admissions from any cause, for diseases of the digestive or respiratory system, or for injuries by weight status at age five.

Results At age 5, 11.6\% and 6.8\% of children were overweight or obese respectively, and $37 \%$ had at least one subsequent hospital admission by their 14th birthday. Compared to children of a healthy weight, there was no increase in the rate of all-cause hospital admissions among overweight or obese children (confounder-adjusted rate ratio (RR), 95\% confidence interval $(\mathrm{CI}): 0.9(0.7,1.1)$ and $1.2(0.9,1.5)$, respectively), or with diseases of the digestive or respiratory system or with injuries.

Conclusion This is to our knowledge the first study to link childhood weight status to hospital admissions in a nationally representative cohort. We found that clinical obesity at primary school entry is not associated with a higher rate of subsequent hospital admissions up to age 14 years in children born in 2000/2001. Future analyses will examine primary care consultations and accident and emergency attendances. Longerterm follow up is also needed to determine whether rates increase with age. 